\title{
Erratum
}

\section{Erratum. Deletion of ATF4 in AgRP Neurons Promotes Fat Loss Mainly via Increasing Energy Expenditure. Diabetes} 2017;66:640-650

Jiali Deng, Feixiang Yuan, Yajie Guo, Yuzhong Xiao, Yuguo Niu, Yalan Deng, Xiao Han, Youfei Guan, Shanghai Chen, and Feifan Guo

https://doi.org/10.2337/db17-er12a

In Fig. $6 E$ of the article cited above, the control panel for Ad-FOXO1 partly duplicates the control panel for Ad-GFP. A revised figure showing the correct control panel for Ad-FOXO1 is shown below. The authors apologize for any inconvenience caused to the readers.

The online version has been updated to correct this error.

E

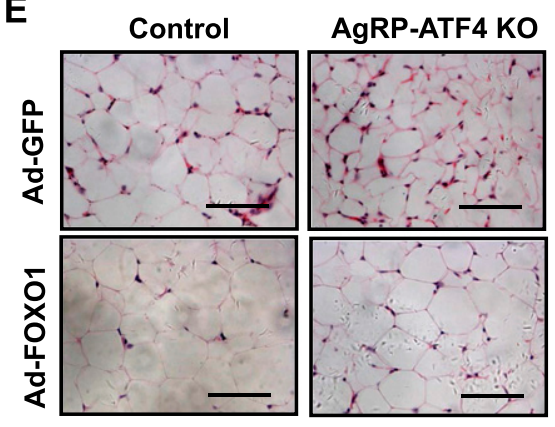

